\title{
Short-Term Impact of a Combined Nutraceutical on Cognitive Function, Perceived Stress and Depression in Young Elderly with Cognitive Impairment: A Pilot, Double-Blind, Randomized Clinical Trial
}

\author{
A.F. Cicero ${ }^{1}$, M. Bove ${ }^{1}$, A. Colletti ${ }^{1}$, M. Rizzo ${ }^{2}$, F. Fogacci ${ }^{1}$, M. Giovannini ${ }^{1}$, C. Borghi ${ }^{1}$

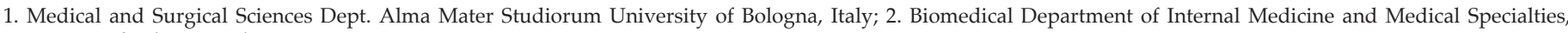 \\ University of Palermo, Italy

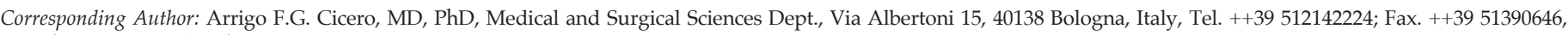 \\ e-mail: arrigo.cicero@unibo.it \\ J Prev Alz Dis 2017;4(1):12-15 \\ Published online August 23, 2016, http:/ / dx.doi.org/10.14283/jpad.2016.110
}

\begin{abstract}
BACKGROUND: The prevalence of senile dementia is increasing worldwide, especially in the developed countries. Nevertheless, drug therapy isn't often enough to treat this condition. Researchers are evaluating the possible impact of a preventive approach, based on an improvement of lifestyle and the intake of micronutrients. Moreover, there is an increasing interest for combined nutraceuticals that can act as memory and learning enhancers, with a significant and beneficial potential on the cognitive disorders.

OBJECTIVE: To evaluate the effects of a rational assemblage of nutraceuticals on cognitive functions in a sample of 30 elderly subjects.

DESIGN: Double bind, cross-over designed trial versus placebo Setting: outpatient clinical practice.

PARTICIPANTS: 30 elderly subjects with basal Mini-Mental State Examination score between 20 and 27 and self-perceived cognitive decline.

INTERVENTION: Treatment with a combination of nutraceuticals based on Bacopa monnieri, L-theanine, Crocus sativus, copper, folate and vitamins of B and D group. After2 months of treatment or placebo.

MEASUREMENTS: Patients were evaluated with Mini-Mental State Examination (MMSE), Perceived Stress Questionnaire (PSQ) and Index and Self-Rating Depression Scale (SRDS).

RESULTS: MMSE and PSQ Index significantly improved in the active treatment arm, both versus baseline and versus the parallel arm. Both groups experienced a significant improving in the SRDS scores.

CONCLUSIONS: We obtained a good and significant improvement of the cognitive functions tested with the MMSE, PSQ-Index and SRDS score, after 2 months of combined therapy of nutraceuticals. Further confirmation will be needed to verify these observations on the middle and long term in a larger number of subjects.
\end{abstract}

Key words: Clinical trial, cognitive impairment, depression, dietary supplement, elderly.

\section{Introduction}

The evidence base on the prevalence of dementia is expanding rapidly, particularly in countries with low and middle incomes. The prevalence of dementia increased exponentially with age, doubling with every 5.5-year increment in age in North America, Latin America, and Asia Pacific (1). Senile dementia is characterized by a decline of many cognitive abilities, particularly reasoning, memory, perceptual speed and language. Many genetic, nutritional and metabolic risk factors have been identified to cause cognitive impairment in older adults (2). Currently there are several available drugs to treat dementia, but many of these are not well-tolerated and usually not supported by a strong evidence-based medicine. Then lifestyle strategies were identified as an effective alternative treatment to prevent neurodegeneration and vascular complications (3). The use of different nutraceuticals such as Bacopa monnieri, L-theanine and Crocus sativus seems to improve some cognitive function and prevent a worsening of the cognitive impairment (4). Bacopa monnieri has been used for centuries in Ayurvedic medicine, as neural tonic, sedative, anti-epileptic, memory and learning enhancer; it contains several active phytochemical constituents as bacosides $\mathrm{A}$ and B, alkaloids, and saponins (5). Its principle mechanisms of action are related to anti-inflammatory, antioxidant, metal chelation, amyloid and cholinergic effects $(6,7)$. L-theanine is a major amino acid found in green tea, able to enhance cognitive functions and to have a positive effect on relaxing, emotional status and on quality sleep (7). It is well known that L-theanine can facilitate the longer-lasting processes responsible for supporting attention across the timeframe of a difficult task (9). Maybe its mechanism of action is based on the increase of brain serotonin, dopamine and GABA levels (10). Its anti-stress effect is probably due to the inhibition of cortical neuron excitation (11). Crocus sativus L. is a perennial herb member of the Iridaceae family and 


Table 1. Main clinical and laboratory parameters of the enrolled patients
\begin{tabular}{|l|c|c|c|c|c|}
\hline & Active treatment (N. 15) & Placebo (N. 15) & & Active treatment (N. 15) & Placebo (N. 15) \\
\hline Age (years) & $66 \pm 3$ & $65 \pm 4$ & Total Cholesterol (mg/dL) & $198.7 \pm 12.7$ \\
\hline Body Mass Index $\left(\mathrm{kg} / \mathrm{m}^{2}\right)$ & $25.6 \pm 1.2$ & $24.9 \pm 1.3$ & LDL-C (mg/dL) & $120.3 \pm 9.8$ \\
\hline SBP (mmHg) & $134.2 \pm 3.3$ & $135.8 \pm 3.6$ & Triglycerides (mg/dL) & $169.4 \pm 19.6$ & $117.3 \pm 10.1$ \\
DBP (mmHg) & $80.4 \pm 2.3$ & $79.6 \pm 2.5$ & HDL-C (mg/dL) & $45.1 \pm 2.4$ & $153.8 \pm 26.6$ \\
\hline Pulse Pressure (mmHg) & $54.1 \pm 2.0$ & $54.2 \pm 2.3$ & Gamma-GT (U/L) & $22.7 \pm 2.2$ & $21.9 \pm 2.5$ \\
\hline Heart Rate (bpm) & $72.4 \pm 8.3$ & $74.1 \pm 9.7$ & Serum Uric Acid (mg/dL) & $4.3 \pm 1.2$ & $4.4 \pm 1.5$ \\
FPG (mg/dL) & $96.9 \pm 3.9$ & $94.7 \pm 4.1$ & Creatinine (mg/dL) & $0.9 \pm 0.2$ \\
\hline
\end{tabular}

by its dark-red stigmas is obtained saffron. The main chemical compound of saffron is crocin, it is known for antidepressant and anti-inflammatory effects and to improve learning and memory (12). Crocin may antagonize the cognitive deficits caused by neurotoxic agents like streptozocin (13) and it may be considered a potential treatment for neurodegenerative disorders. (14). Its main mechanism of action is based on the inhibition of acetyl-cholinesterase activity and on its antioxidant properties (15). Folate, copper and some vitamins are essential nutrients for development, function and regeneration of nervous systems. Their deficiency has been linked to many neurological disorders, such as cognitive decline, dementia and Alzheimer's disease (16). A significant deficiency of folate can affect central monoamine metabolism and get worse depressive disorders (17). B-vitamins, phospholipids and micronutrients are effective cofactors to enhance the precursors availability to make neuronal membranes and synapses (18), vitamin B6 is moreover involved in the regulation of mental function and mood (19) .

The aim of our study was to test the short-term effect of combined nutraceuticals on cognitive functions, perceived stress and depression in a sample of 30 young elderly with cognitive impairment, to also verify the tolerability and the safety of this product.

\section{Methods}

This is a pilot, double-blind, placebo-controlled, randomized clinical trial with parallel arms. We enrolled 30 young elderly subjects (mean age $66 \pm 3$ ), not affected by cardiovascular diseases nor type 2 diabetes, with MMSE at baseline include between 20 and 27, and selfperceived cognitive decline without known diagnosis of cognitive decline nor dementia.

Exclusion criteria were:

- Previous history of Transient Ischemic Attacks nor strokes

- Uncontrolled blood pressure or LDL-cholesterol values

- Any pharmacological treatment not stabilized from at least 6 months

This research was carried out in accordance with the Declaration of Helsinki of the World Medical Association and all subjects signed an informed consent form. The study was approved by the local .

\section{Ethical Board}

Randomization was done using a drawing of envelopes containing randomization codes prepared by an independent statistician and specific software. The envelopes were then further mixed and distributed to the investigators who assigned the randomization code in a progressive way to the enrolled subjects. A copy of the code was provided only to the person responsible of performing the statistical analysis.

The subjects were randomized to assume a combined nutraceuticals containing Bacopa monnieri dry extract $320 \mathrm{mg}$, L-Teanina $100 \mathrm{mg}$, Crocus sativus $30 \mathrm{mg}$, some vitamins (Vitamin B6 9,5 mg, Biotine $450 \mathrm{mcg}$, Folic acid $400 \mathrm{mcg}$, Vitamin B12 $33 \mathrm{mcg}$, Vitamin D $25 \mathrm{mcg}$ ) and Cupper 2 mg (kindly furnished by Inpha Duemila S.r.l, Lecco, Italy, or an indistinguishable placebo, 1 capsule per day, for 8 weeks.

The following questionnaires have been submitted at the baseline and at the end of the study:

*Mini-Mental State Examination

*Perceived Stress Questionnaire Index

*Self-rating Depression Scale

The tolerability and acceptability of the product have been also investigated at the end of the trial with a semiquantitative scale.

The participants were tested during a morning visit in a quiet room by neuro-psychologically trained research assistants who were not directly involved in the study. Cognitive testing was assessed by the age and education adjusted mini-mental state examination (MMSE), a widely used screening tool for cognitive impairment that covers five areas of cognitive function including orientation, attention, calculus, recall and language with a score ranging from 0 to 30 . The value of 27 of MMSE score has been considered as normality threshold, as it is commonly used to indicate normal cognitive function, (20). Then the Perceived Stress Questionnaire Index (21) and the Self-rating Depression Scale (22) were also administered to the same subjects at the beginning and at the end of the study. 


\begin{tabular}{|c|c|c|c|c|}
\hline & \multicolumn{2}{|l|}{ Active } & \multicolumn{2}{|l|}{ Placebo } \\
\hline & Pre-treatment & Post-treatment & Pre-treatment & Post-treatment \\
\hline MMSE & $23.1 \pm 0.9$ & $24.5 \pm 1.0^{* \circ}$ & $23.2 \pm 1.1$ & $23.1 \pm 0.9$ \\
\hline PSQ Index & $2.7 \pm 0.4$ & $2.2 \pm 0.7^{* \circ}$ & $2.6 \pm 0.8$ & $2.4 \pm 0.9$ \\
\hline SRDS & $42.8 \pm 8.4$ & $37.1 \pm 7.6^{*}$ & $43.6 \pm 9.3$ & $40.9 \pm 8.8^{*}$ \\
\hline
\end{tabular}

${ }^{*} \mathrm{P}<0.05$ Vs. baseline ; ${ }^{\circ} \mathrm{P}<0.05 \mathrm{Vs}$. placebo

Throughout the study, we instructed patients to take the new product first dose on the day after they were given the study product in a blinded box. At the same time, all unused products were retrieved for inventory. Product compliance was assessed by counting the number of product doses returned at the time of specified clinic visits.

Data have been analyzed using intention to treat by mean of the Statistical Package for Social Science (SPSS) 21.0, version for Windows. Normally distributed baseline characteristics of the population have been compared using Student's t test, not normally ones using the Mann-Whitney-U test. The questionnaire scores have been compared by the Wilcoxon rank test. All data are expressed as means and SD. A p level of $<0.05$ was considered significant for all tests.

\section{Results}

At the baseline, the two patient groups were comparable as it regards the main clinical and laboratory parameters (table 1). Moreover, all patients were treated with ACE-inhibitors/sartans and statins.

All enrolled subjects completed the study. Only one patient claimed aftertaste after active product intake. Compliance to the products was not different between the subjects groups: $95 \%$ active product, $90 \%$ placebo.

On a graded scale (1-10): the mean acceptability was 9 for both Active product and Placebo

The main results of the trials are resumed in table 2. MMSE and PSQ Index significantly improved in the active treatment arm, both versus baseline and versus the parallel arm. Both groups experienced a significant improving in the SRDS score.

\section{Discussion}

Dementia is a pathological condition considered an health- and social-care priority for many high-income countries, that focused a large part of their economic resources on prevention (23). As stated above (3), lifestyle strategies were identified as an effective and alternative treatment to prevent neuro-degeneration and vascular complications. Several studies recently suggest that a Mediterranean diet, an antioxidant-rich cardioprotective dietary pattern, delays mild cognitive impairment and Alzheimer's disease $(24,25)$. Thus, it is plausible that antioxidant-rich foods might afford protection from cognitive decline and major neurodegenerative diseases (26). Our research would emphasize the potential use of combined nutraceuticals, such as Bacopa monnieri, L-theanine and Crocus sativus in age-related diseases treatment. The principal biological mechanism of these natural products are related to anti-inflammatory, antioxidant, anti-stress, metal chelation, amyloid and cholinergic effects $(6,7)$. Rastogi et al. suggest that a longterm $(200 \mathrm{mg} / \mathrm{kg}$ orally per day for 3 months) bacoside administration can lead to a significant decreases in proinflammatory cytokines (interleukin-1b, tumor necrosis factor-alpha but not interferon-c), a significant induction of inducible nitric oxide synthetase (iNOS) expression, and a significant reduction of total nitrite and lipofuscin content in the cortex (27). Changes in brain alpha oscillatory activity using electroencephalography (EEG) have been studied as correlates of L-theanine potential anti-stress effects, using resting state recordings, and cognitive enhancing effects, using task-related recordings (28). Evidence from recent in vitro and in vivo research indicates that saffron has potential anticancerogenic (cancer-suppressing), anti-mutagenic (mutation-preventing), antioxidant, antidepressant, and memory-enhancing properties (29). All of these properties have allowed us to test an original and innovative composition of these natural substances in a sample of young elderly subjects, to evaluate the possible improvement of their main cognitive functions, perceived stress and depression rate. The results obtained demonstrate the effectiveness and the safety of this combination therapy. The synergistic action of Bacopa monnieri, L-theanine, Crocus sativus, copper, folate and vitamins of B and D group after 2 months of treatment resulted in a significant improvement of cognitive decline, perceived stress and Depression tested with MMSE, PSQ-Index and SRDS scores.

Our study has also some relevant limitation. In particular, the patient sample is small, and the study duration relatively short, so that we cannot predict if the observed results will be maintained on a longer followup. However, this is a preliminary trial, and the crossover design reinforce our observation. Then, the results are encouraging to perform new clinical trials on a larger number of subjects with a longer duration of treatment. If our results will be also confirmed by different future studies this combined nutraceutical could be considered 
a rational and alternative therapeutic option for minor cognitive disorders, being relatively inexpensive and well-tolerated.

Funding: This study has been supported with institutional funding from the University of Bologna.

Conflict of Interest: No one authors has a direct conflict of interest in the publication of this paper.

Ethical Standards: The study has been carried out in agreement with the Declaration of Helsinki. All the enrolled subjects were asked to sign an informed consent.

\section{References}

1. Prince M, Bryce R, Albanese E, Wimo A, Ribeiro W, Ferri CP. The global prevalence of dementia: a systematic review and metaanalysis. Alzheimers Dement 2013;9(1):63-75. doi: 10.1016/j.jalz.2012.11.007

2. Flirski M, Sobow T. Biochemical markers and risk factors of Alzheimer's disease. Curr Alzheimer Res 2005;2:47-64

3. Polidori MC, Schulz RJ. Nutritional contributions to dementia prevention: main issues on antioxidant micronutrients. Genes Nutr 2014;9(2):382. doi: 10.1007/s12263-013-0382-2

4. Mecocci P, Tinarelli C, Schulz RJ, Polidori MC. Nutraceuticals in cognitive impairment and Alzheimer's disease. Front Pharmacol 2014;5:147. doi: 10.3389/fphar.2014.00147

5. Sivaramakrishna C, Rao CV, Trimurtulu G, Vanisree M, Subbaraju GV. Triterpenoid glycosides from Bacopa monnieri. Phytochemistry 2005;66(23):2719-2728

6. Stough C, Singh H, Zangara A. Mechanisms, Efficacy, and Safety of Bacopa monnieri (Brahmi) for Cognitive and Brain Enhancement. Evid Based Complement Alternat Med 2015:717605. doi: 10.1155/2015/717605

7. Aguiar S, Borowski T. Neuropharmacological review of the nootropic herb Bacopa monnieri. Rejuvenation Res 2013;16(4):313-26. doi: 10.1089/ rej.2013.1431

8. Türközü D, Şanlier N, L-Theanin. Unique Aminoacid of Tea, and Its Metabolism, Health Effects, Safety. Crit Rev Food Sci Nutr 2015;20:0. doi:10.1 080/10408398.2015.1016141

9. Gomez-Ramirez M, Kelly SP, Montesi JL, Foxe JJ. The effects of L-theanine on alpha-band oscillatory brain activity during a visuo-spatial attention task. Brain Topogr 2009;2(1):44-51. doi: 10.1007/s10548-008-0068-z

10. Nathan PJ, Lu K, Gray M, Oliver C. The neuropharmacology of L-theanine(N-ethyl-L-glutamine): a possible neuroprotective and cognitive enhancing agent. J Herb Pharmacother 2006;6(2):21-30

11. Kimura K, Ozeki M, Juneja LR, Ohira H. L-Theanine reduces psychological and physiological stress responses. Biol Psycho 2007;74(1):39-45

12. Schmidt M, Betti G, Hensel A (2007) Saffron in phytotherapy: pharmacology and clinical uses. Wien Med Wochenschr 2007;157(13-14):315-9

13. Naghizadeh B, Mansouri MT, Ghorbanzadeh B, Farbood Y, Sarkaki A. Protective effects of oral crocin against intracerebroventricular streptozotocin-induced spatial memory deficit and oxidative stress in rats.
Phytomedicine 2013;20(6):537-42. doi: 10.1016/j.phymed.2012.12.019

14. Akhondzadeh S, Sabet MS, Harirchian MH, Togha M, Cheraghmakani H, Razeghi S, Hejazi SSh, Yousefi MH, Alimardani R, Jamshidi A, Zare F, Moradi A. Saffron in the treatment of patients with mild to moderate Alzheimer's disease: a 16-week, randomized and placebo-controlled trial. J Clin Pharm Ther 2010;35(5):581-8. doi: 10.1111/j.1365-2710.2009.01133.x

15. Pitsikas N. The Effect of Crocus sativus L. and Its Constituents on Memory: Basic Studies and Clinical Applications. Evid Based Complement Alternat Med 2015:926284. doi: 10.1155/2015/926284.

16. Tucker KL. Nutrient intake, nutritional status, and cognitive function with aging. Ann N Y Acad Sci. 2016 Mar;1367(1):38-49. doi: 10.1111/nyas.13062.

17. Horvat P, Gardiner J, Kubinova R, Pajak A, Tamosiunas A, Schöttker B, Pikhart H, Peasey A, Jansen E, Bobak M. Serum folate, vitamin B-12 and cognitive function in middle and older age: The HAPIEE study. Exp Gerontol. 2016 Apr;76:33-8. doi: 10.1016/j.exger.2016.01.011.

18. Engelborghs S, Gilles C, Ivanoiu A, Vandewoude M. Rationale and clinica data supporting nutritional intervention in Alzheimer's disease. Acta Clin Belg 2014;69(1):17-24. doi: 10.1179/0001551213Z.0000000006.

19. Smith PJ, Blumenthal JA. Dietary Factors and Cognitive Decline. J Prev Alzheimers Dis. 2016 Mar;3(1):53-64.

20. Crum RM, Anthony JC, Bassett SS, Folstein MF. Population-based norms for the mini-mental state examination by age and educational level. JAMA 1993;269:2386-2391

21. Fliege H, Rose M, Arck P, Walter OB, Kocalevent RD, Weber C, Klapp BF. The Perceived Stress Questionnaire (PSQ) reconsidered: validation and reference values from different clinical and healthy adult samples. Psychosom Med 2005;67(1):78-88

22. Zung WW. A self-rating depression scale. Arch Gen Psych 1965;12:63-70

23. Schaller S, Mauskopf J, Kriza C, Wahlster P, Kolominsky-Rabas PL. The main cost drivers in dementia: a systematic review. Int J Geriatr Psychiatry 2015;30(2):111-129. doi: 10.1002/gps.4198

24. Psaltopoulou T, Sergentanis TN, Panagiotakos DB, Sergentanis IN, Kost $\mathrm{R}$, Scarmeas N. Mediterranean diet, stroke, cognitive impairment, and depression: A meta-analysis. Ann Neurol 2013;74(4):580-91. doi: 10.1002/ ana.23944

25. Singh B, Parsaik AK, Mielke MM, Erwin PJ, Knopman DS, Petersen $\mathrm{RC}$, Roberts RO. Association of mediterranean diet with mild cognitive impairment and Alzheimer's disease: a systematic review and meta-analysis. J Alzheimers Dis 2014;39(2):271-82. doi: 10.3233/JAD-130830

26. Von Bernhardi R, Eugenín J. Alzheimer's disease: redox dysregulation as a common denominator for diverse pathogenic mechanisms. Antioxid Redox Signal 2012;16(9):974-1031. doi: 10.1089/ars.2011.4082

27. Rastogi M, Ojha R, Prabu PC, Devi DP, Agrawal A, Dubey GP. Amelioration of age associated neuroinflammation on long term bacosides treatment. Neurochem Res 2012;37:869-874. doi: 10.1007/s11064-011-0681-1

28. Juneja LR, Chu DC, Okubo T, Nagato Y, Yokogoshi H. L-theanine-A unique amino acid of green tea and its relaxation effect in humans. Trends Food Sci Technol 1999:10:199-204

29. Bathaie SZ, Mousavi SZ. New applications and mechanisms of action of saffron and its important ingredients. Crit Rev Food Sci Nutr 2010;50(8):761786. doi: $10.1080 / 10408390902773003$ 